\title{
Galaxy Interactions and Their Cosmic and Morphological Evolution
}

\author{
Tapan K. Chatterjee \\ University of the Americas (Department of Physics), A. P. 1316, Puebla, Mexico \\ e-mail:chtapan@yahoo.com
}

Merger theory, in its extreme form, postulates that ellipticals are the products of mergers of spirals. While the morphology-clustercentric relationship (Whitmore et al., 1993), indicates that the morphological fractions are unaffected by the local properties of the clusters, considerable observational evidence has surfaced in favor of the existence of marginally bound pairs; that are the source of the most frequent collisions (Chengalur et al., 1996 and references therein). To test the global impact of galaxy collisions on their evolution we have studied many collisions between spirals and ellipticals, as well as spiral and elliptical pairs. The details of the models and methods are in Chatterjee \& Magalinsky, 2002; and we refer to it. Marginally bound, eccentric orbits are found to maintain their eccentricities for a long time; which is supported by analytical research (Magalinsky \& Chatterjee, 2000). The calculation of collision frequency indicates that only of the order of $5 \%$ of ellipticals can be formed in this way. A remarkable phenomenon is that the fraction of blue galaxies around $\mathrm{z}=0.3$ to 0.5 is more than in the present epoch - the B-O effect (Butcher \& Oemler, 1978). While observations indicate that the effect is primarily due to an increase in galaxies with perturbed morphology (e.g., Faber et al., 1995), in the same environment we find a homogeneous population of normal red galaxies, that have almost finished their star formation. Evidently the passive evolution of elliptical galaxies is correct only to the first order and involves secondary star formation; due to interactions, but not necessarily mergers. This supports the observational evidence that ellipticals undergo a passive luminosity evolution, in which the main star formation episode occurs at a high redshift $\mathrm{z}>2$ and the assembly of the galaxies is almost complete by z $=1$; (e. g., Aragon-Salamanca et al., 1993, Lilly et al., 1995; van Dokkum \& Franx, 1996; De Propris et al., 1999); Broadhurst \& Bouwens, 2000). Spirals are likely to have formed afterwards. Galaxy collisions are sporadic phenomena, which bestow specific peculiarities; like in BCMs (e.g., Dressler, 1984; c.f., Combes, 2006).

\section{References}

Aragon-Salamanca, A., Ellis, R. S., Couch, W. J., \& Carter, D. 1993, MNRAS, 262, 764.

Broadhurst, T., \& Bouwens, R. J., 2000, ApJ, 530, L53.

Butcher, H., \& Oemler, A., 1978, ApJ, 219, 18.

Chatterjee, T. K., \& Magalinsky, V. B. 2002, Astron. Astrophys. Trans. 21, 79.

Chengalur, J., Salpeter, E. E., \& Terzian, Y. 1966, ApJ, 461, 546.

Combes, F. 2006, this symposium.

De Propris, R., Stranford, S. A., Eisenhardt, P. R., Dickson, M., \& Elston, R. 1999, ApJ, 118, 719.

Dressler, A. 1984, ARA\&A, 22, 185.

Faber, S. M., Trager, S. C., Gonzalez, J. J., \& Worthy, G. 1995, In IAU Symp. 164 (Reidel).

Lilly, S. J., Tresse, L., Hammer, F., Crampton, D., \& LeFevre, O. 1995, ApJ 455, 108.

Magalinsky, V. B., \& Chatterjee, T. K. 2000, Astron. Astrophys. Trans. 18, 807.

Van Dokkum, P. G., \& Franx, M. 1966, MNRAS, 281, 985.

Whitmore, B. C., Gilmore, D. M., \& Jones, C., 1993, ApJ, 407, 489. 